\title{
Seismic moment and volume change of a spherical source
}

\author{
Hiroyuki Kumagai ${ }^{1 *}$, Yuta Maeda ${ }^{1}$, Mie Ichihara² ${ }^{2}$ Nobuki Kame ${ }^{2}$ and Tetsuya Kusakabe ${ }^{2}$
}

\begin{abstract}
A spherical source, one of the simplest seismic sources, has been represented in various ways in the literature. These representations include a spherical source with outward radial expansion (S1), a spherical crack source with outward and inward crack wall motions along the spherical surface (S2), an isotropic source represented by three mutually perpendicular vector dipoles (S3) or three mutually perpendicular tensile cracks (S4), and a spherical source undergoing a transformational expansion (S5). We systematically examined these sources and their static displacement fields to clarify how these representations are mutually related. We also considered the sources in a bimaterial medium, in which the source material is different from the surrounding medium, as a model of a magma or hydrothermal reservoir. Our examinations show that the source volume change of a spherical source (S1) (actual volume) can be uniquely determined from the seismic moment of an isotropic source (S3) regardless of our assumption of the source medium and that the actual volume of S1 is related to the seismic moment of S3 through the equivalence of the displacement fields due to these two sources. The seismic moment of S3 is also related through another equation to the source volume change of three tensile cracks (S4), which is equal to the source volume changes defined in S2 and S5. This relation has different forms, depending on the source medium and source process. This study provides a unified view for quantifying a spherical source using the seismic moment of an isotropic source determined from waveform inversion analysis.
\end{abstract}

Keywords: Volume source; Isotropic source; Source representation; Volcano seismology; Waveform inversion

\section{Background}

Spherical and cylindrical volume changes have been considered as sources of volcano seismic signals (e.g., Kanamori et al. 1980; Fukuyama and Takeo 1987; Uhira and Takeo 1994; Dreger et al. 2000; Legrand et al. 2000; Tameguri et al. 2002; Ohminato et al. 2006; Maeda and Takeo 2011; Kumagai et al. 2011). Although a spherical source is one of the simplest seismic sources, there are various ways to represent it. These representations include a spherical source with outward radial expansion (e.g., Mogi 1958) (S1, Figure 1a), a spherical crack source of Müller (2001) (S2, Figure 1b), an isotropic source represented by three mutually perpendicular vector dipoles (S3, Figure 1c) or three mutually perpendicular tensile cracks (S4, Figure 1d), and a spherical source undergoing a trans-

\footnotetext{
*Correspondence: kumagai@eps.nagoya-u.ac.jp

1 Graduate School of Environmental Studies, Nagoya University, Furo-cho,

Chikusa-ku, Nagoya 464-8601, Japan

Full list of author information is available at the end of the article
}

formational expansion based on the concept of imaginary cutting, straining, and welding operations of Eshelby (1957) as introduced by Aki and richards (2002), which we call an Eshelby spherical source (S5, Figure 2a).

Using these representations, the seismic moment of a spherical source has been defined in two different ways (Müller 2001; Richards and Kim 2005). The two definitions are

$$
\begin{aligned}
& M_{0}=(\lambda+2 \mu) \Delta V_{\mathrm{s}}, \\
& M_{0}=(\lambda+2 \mu / 3) \Delta V_{\mathrm{f}},
\end{aligned}
$$

where $\lambda$ and $\mu$ are Lamé's constants and $\Delta V_{\mathrm{s}}$ and $\Delta V_{\mathrm{f}}$ are two different definitions of source volume changes (see below). The recent volcano seismology literature has used Equation 1 (e.g., Kawakatsu and Yamamoto 2007; Kumagai 2009; Chouet 2013), whereas earlier studies generally used Equation 2 (e.g., Chouet 1996; Julian et al. 1998; Dreger et al. 2000; Nishimura and Iguchi 2011).

According to Kawakatsu and Yamamoto (2007), the volume change in Equation $2\left(\Delta V_{\mathrm{f}}\right)$ corresponds to the

\section{Springer}

(c) 2014 Kumagai et al: licensee Springer. This is an Open Access article distributed under the terms of the Creative Commons Attribution License (http://creativecommons.org/licenses/by/2.0), which permits unrestricted use, distribution, and reproduction in any medium, provided the original work is properly credited. 
(a) $\mathrm{S} 1$

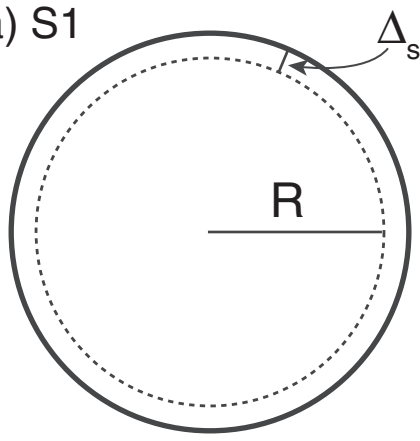

(c) S3

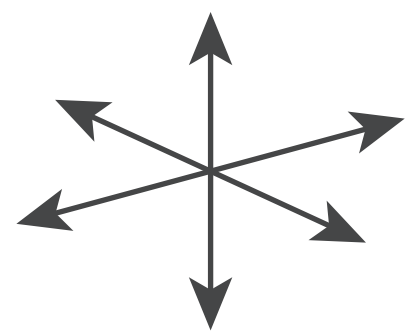

(b) $\mathrm{S} 2$

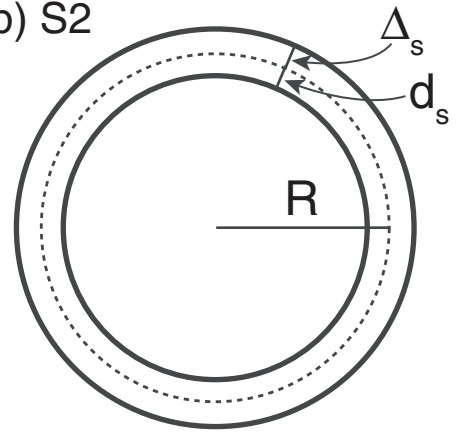

(d) S4

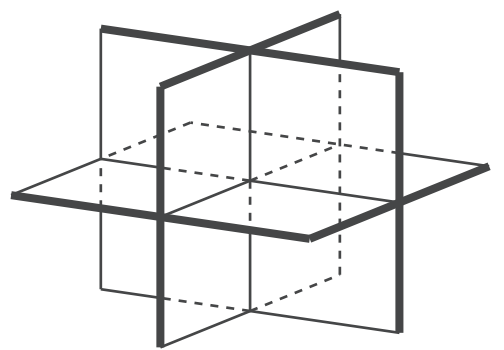

Figure 1 Source representations. (a) A spherical source with purely outward radial expansion of $\Delta_{S}(S 1)$, (b) a spherical crack source with outward and inward displacements ( $\Delta_{\mathrm{S}}$ and $d_{\mathrm{s}}$, respectively) along the spherical surface (S2), (c) an isotropic source with three mutually perpendicular vector dipoles (S3), and (d) a superposition of three mutually perpendicular tensile cracks (S4).

stress-free volume, introduced by Eshelby (1957), whereas the actual volume in Equation $1\left(\Delta V_{\mathrm{s}}\right)$ may be smaller than the stress-free volume because of the confining pressure of the surrounding medium. Kawakatsu and Yamamoto (2007) also noted that the actual volume corresponds to the volume change used in the Mogi model (e.g., Mogi 1958).

Müller (2001) noted that the discrepancy between Equations 1 and 2 could not be resolved, and he interpreted these two forms to correspond to the limits of the actual volume of the source. Wielandt (2003) discussed the discrepancy and noted that the source volume change depends on the source geometry. Richards and Kim (2005) argued that each relationship is based on a different definition of volume changes at the source and that Equation 1 is preferred for characterizing underground explosions.

It is not clear why a spherical source has been represented in different ways and how these representations, including the two seismic moment equations, are mutually related. In Eshelby's operations and Müller's spherical crack, the source material is assumed to be the same as the surrounding medium. In volcanic regions, sources may be filled with magmatic or hydrothermal fluids. No examinations have been made on a spherical source in such bimaterial media, although the source representation of fault slip on a bimaterial interface has been discussed in various studies (e.g., Ampuero and Dahlen 2005).

In this paper, we first review the spherical source representations mentioned above and then derive the static displacement fields due to these sources in an infinite medium and in a half-space. We compare the analytical forms of these displacements to clarify the relationships among these representations. We further examine a spherical source in a bimaterial medium, which is of fundamental importance as a model of a magma or hydrothermal reservoir.

\section{Methods}

\section{Source representations}

Müller (2001) proposed to define a spherical source in terms of the outward crack wall motion of a spherical crack. Let us first define a spherical crack source (S2). We consider the moment density tensor of a tensile crack with normal direction $(\theta, \phi)$ (Figure 3a):

$$
\boldsymbol{m}(\theta, \phi)=D_{\mathrm{s}}\left(\begin{array}{rrr}
\lambda+2 \mu \sin ^{2} \theta \cos ^{2} \phi & 2 \mu \sin ^{2} \theta \sin \phi \cos \phi & 2 \mu \sin \theta \cos \theta \cos \phi \\
2 \mu \sin ^{2} \theta \sin \phi \cos \phi & \lambda+2 \mu \sin ^{2} \theta \sin ^{2} \phi & 2 \mu \sin \theta \cos \theta \sin \phi \\
2 \mu \sin \theta \cos \theta \cos \phi & 2 \mu \sin \theta \cos \theta \sin \phi & \lambda+2 \mu \cos ^{2} \theta
\end{array}\right),
$$



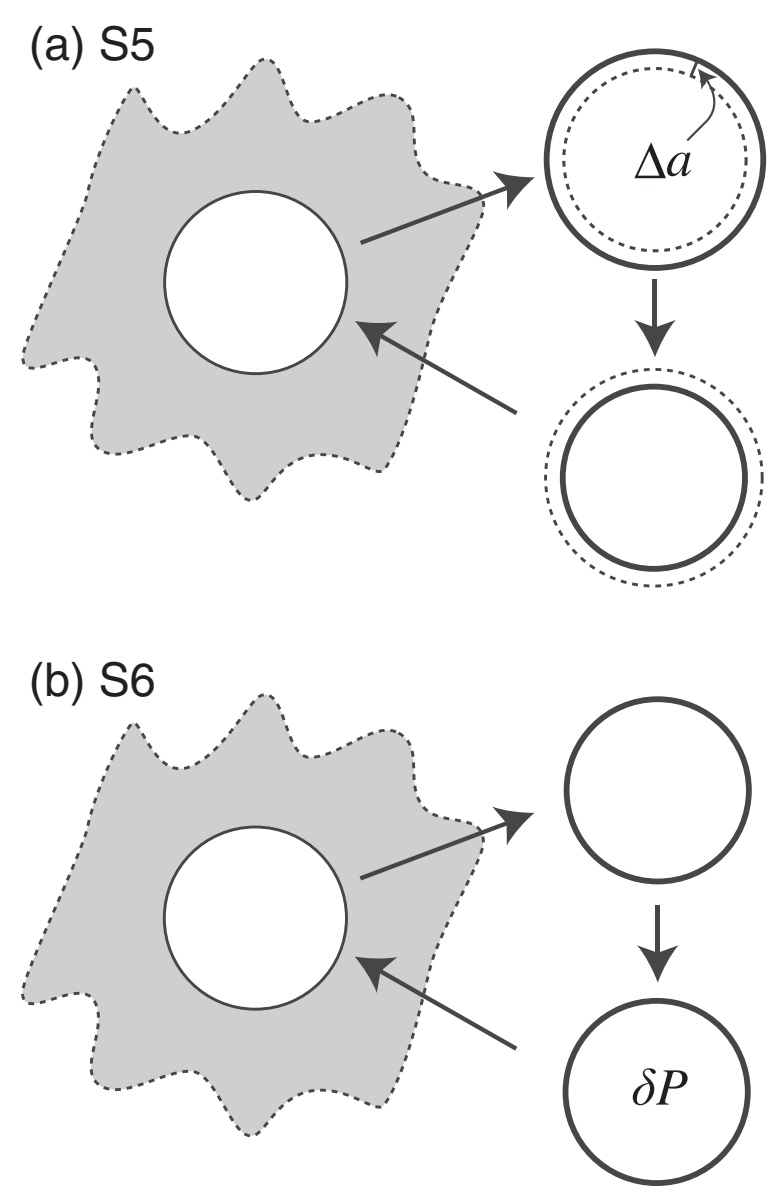

Figure 2 Spherical source with stress-free strain and strain-free stress. (a) Schematic view of a spherical source undergoing a transformational expansion (S5) based on imaginary cutting, straining, and welding operations of Eshelby (1957). (b) A spherical source in which a strain-free stress or pressure $(\delta P)$ is applied to the removed source volume (S6) (see text for details).

where $D_{\mathrm{s}}$ is the displacement discontinuity of a tensile crack (e.g., Chouet 1996). We consider a spherical crack surface with radius $R$ where a constant radial displacement discontinuity $D_{\mathrm{s}}=\left(d_{\mathrm{s}}+\Delta_{\mathrm{s}}\right)$ occurs (Figure $\left.1 \mathrm{~b}\right)$. Here, the inner wall of the crack moves inward by $d_{\mathrm{s}}$, and the outer wall moves outward by $\Delta_{\mathrm{s}}$. The moment tensor of the spherical crack source can be obtained by integrating the moment density tensor of the tensile crack over the surface with radius $R$ (Figure $1 \mathrm{~b}$ ):

$$
\begin{aligned}
\boldsymbol{M} & =\int_{0}^{2 \pi} \int_{0}^{\pi} \boldsymbol{m}(\theta, \phi) R^{2} \sin \theta d \theta d \phi \\
& =\Delta V_{\mathrm{D}}\left(\begin{array}{ccc}
\lambda+2 \mu / 3 & 0 & 0 \\
0 & \lambda+2 \mu / 3 & 0 \\
0 & 0 & \lambda+2 \mu / 3
\end{array}\right),
\end{aligned}
$$

where $\Delta V_{\mathrm{D}}$ is the volume given as

$$
\Delta V_{\mathrm{D}}=4 \pi R^{2} D_{\mathrm{s}}=4 \pi R^{2}\left(d_{\mathrm{S}}+\Delta_{\mathrm{s}}\right) .
$$

By solving the elastostatic boundary-value problem (see Subsection 'Displacement fields'), we obtain

$$
\frac{\Delta V_{\mathrm{s}}}{\Delta V_{\mathrm{D}}}=\frac{\lambda+2 \mu / 3}{\lambda+2 \mu},
$$

where $\Delta V_{\mathrm{s}}=4 \pi R^{2} \Delta_{\mathrm{s}}$.

An isotropic source is defined as three mutually perpendicular vector dipoles (S3, Figure 1c), of which the moment tensor is given as

$$
\boldsymbol{M}=M_{0}^{\text {Iso }}\left(\begin{array}{lll}
1 & 0 & 0 \\
0 & 1 & 0 \\
0 & 0 & 1
\end{array}\right),
$$

where $M_{0}^{\text {Iso }}$ is the seismic moment for an isotropic source. The three mutually perpendicular vector dipoles can be represented as the superposition of three mutually perpendicular tensile cracks (S4, Figure 1d). In such a case, $M_{0}^{\text {Iso }}$ can be defined as

$$
M_{0}^{\text {Iso }}=(\lambda+2 \mu / 3) \Delta V_{\mathrm{i}} .
$$




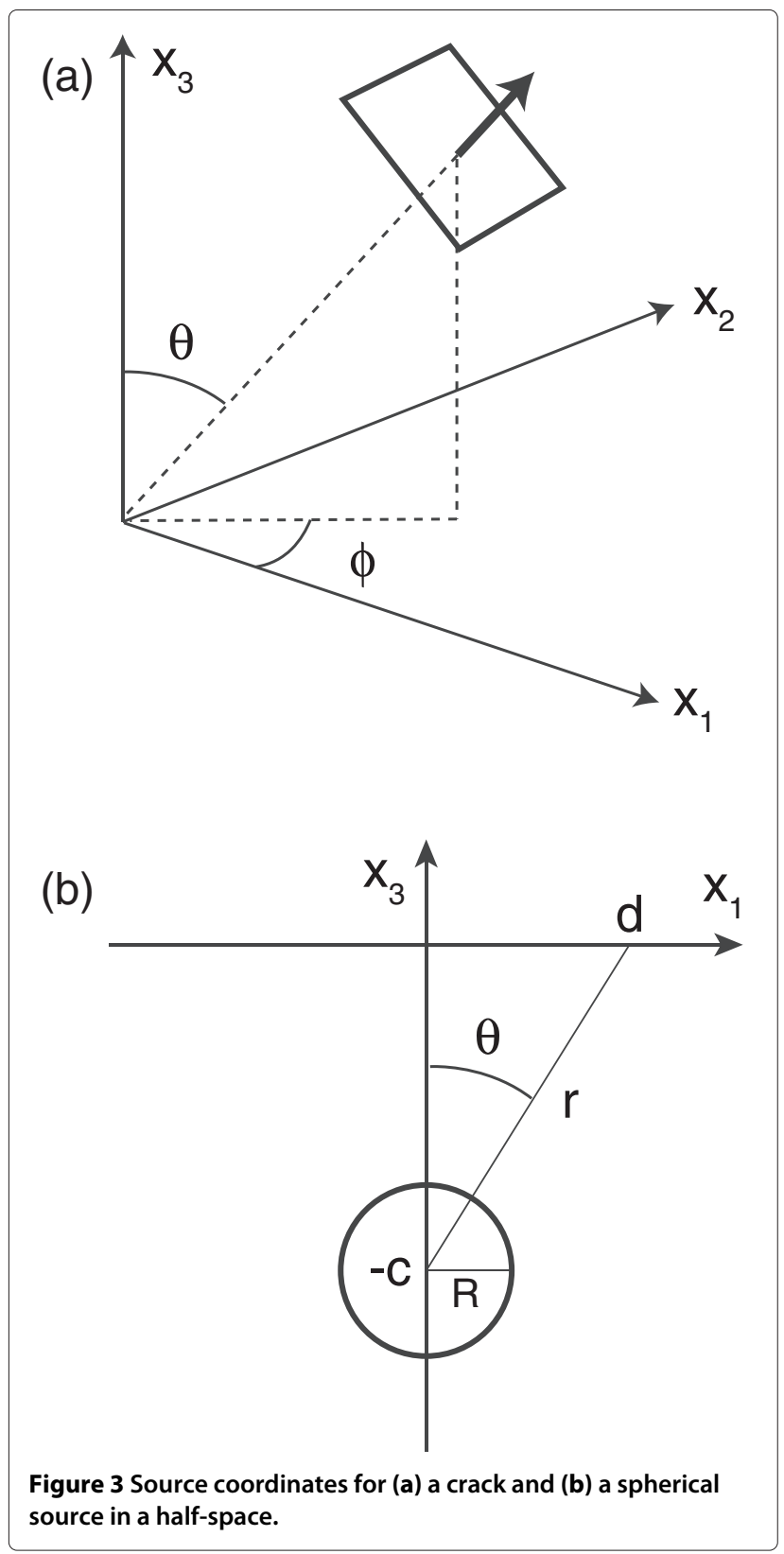

Here, $\Delta V_{\mathrm{i}}=3 \Delta V_{\mathrm{c}}$, where $\Delta V_{\mathrm{c}}$ is the incremental change in the volume of each crack.

Finally, we consider an Eshelby spherical source following Aki and richards (2002) (S5, Figure 2a). A spherical volume with radius $R$ removed from its surroundings expands to $R+\Delta a$ with stress-free volumetric strain. We apply the surface traction that will restore the volume source to its original radius $R$, put the volume back in its hole, and weld the material across the cut. We release the applied traction, which results in the source volume expanding to $R+\Delta_{\mathrm{s}}$. The volume defined as $\Delta V_{\mathrm{f}}=$ $4 \pi R^{2} \Delta a$ is the stress-free volume, and the volume defined as $\Delta V_{\mathrm{S}}=4 \pi R^{2} \Delta_{\mathrm{S}}$ is the actual volume or Mogi volume. We define the seismic moment using the stress-free volume as

$$
M_{0}=(\lambda+2 \mu / 3) \Delta V_{\mathrm{f}},
$$

and the seismic moment using the Mogi volume may be given as

$$
M_{0}=(\lambda+2 \mu) \Delta V_{\mathrm{s}}
$$

\section{Displacement fields}

Let us consider the static displacement fields due to each of the described sources. For the spherical source (S1), spherical crack source (S2), and Eshelby spherical source (S5), the displacement fields in an infinite medium are described by the following static equilibrium equation:

$$
\frac{\partial^{2} u_{\mathrm{r}}}{\partial r^{2}}+\frac{2}{r} \frac{\partial u_{\mathrm{r}}}{\partial r}-\frac{2}{r^{2}} u_{\mathrm{r}}=0,
$$

where $u_{\mathrm{r}}$ is the radial displacement. We denote the regions inside and outside the sphere with radius $R$ as regions 1 and 2, respectively. Equation 11 has two solutions: $u_{\mathrm{r}}=A r$ and $u_{\mathrm{r}}=B / r^{2}$, where $A$ and $B$ are constants. The former is the interior solution for region $1(r \leq R)$, and the latter is the exterior solution for region $2(r \geq R)$.

\section{Spherical source}

We first consider the spherical source (S1). If the pressure in the spherical source increases by $\Delta P$, the spherical source surface moves outward by $\Delta_{\mathrm{S}}$ at $r=R$. Then, the boundary conditions at $r=R$ are given as

$$
\begin{aligned}
& u_{\mathrm{r}}^{e}=\Delta_{\mathrm{s}} \\
& \sigma_{\mathrm{rr}}^{e}=-\Delta P,
\end{aligned}
$$

where $u_{\mathrm{r}}^{e}$ and $\sigma_{\mathrm{rr}}^{e}$ are the radial displacement and radial stress in region 2, respectively. Using these boundary conditions and the exterior solution $u_{\mathrm{r}}=B / r^{2}$, we find that

$$
\Delta P=4 \mu \frac{\Delta_{\mathrm{s}}}{R},
$$

and

$$
u_{\mathrm{r}}^{e}=\frac{\Delta V_{\mathrm{s}}}{4 \pi r^{2}},
$$

where $\Delta V_{\mathrm{s}}=4 \pi R^{2} \Delta_{\mathrm{s}}$.

Following Sezawa (1931), we consider the spherical source in a half-space (Figure $3 \mathrm{~b}$ ). The radial displacement on the free surface at distance $r$ is given as

$$
\begin{aligned}
u_{\mathrm{r}}^{e} & =\frac{(\lambda+2 \mu)}{2 \mu(\lambda+\mu)} \frac{R^{3} \Delta P}{r^{2}}, \\
& =\frac{(\lambda+2 \mu)}{2 \pi(\lambda+\mu)} \frac{\Delta V_{\mathrm{s}}}{r^{2}} .
\end{aligned}
$$


If we assume $\lambda=\mu$, the vertical and radial components for $u_{\mathrm{r}}^{e}$ in cylindrical coordinates are given as

$$
\begin{aligned}
& u_{\mathrm{r}}^{e} \cos \theta=\frac{3 R^{3} \Delta P}{4 \mu} \frac{c}{\left(d^{2}+c^{2}\right)^{3 / 2}}, \\
& u_{\mathrm{r}}^{e} \sin \theta=\frac{3 R^{3} \Delta P}{4 \mu} \frac{d}{\left(d^{2}+c^{2}\right)^{3 / 2}},
\end{aligned}
$$

respectively, where $c$ is the source depth and $d$ is the horizontal source distance (Figure $3 \mathrm{~b}$ ). Equations 18 and 19 represent the displacement field of the Mogi model (Mogi 1958).

Comparing Equation 17 for a half-space with Equation 15 for an infinite medium, we find that these two equations differ by a factor of $2(\lambda+2 \mu) /(\lambda+\mu)$ (Figure $4 a$ ). For this consideration, we used $\Delta V_{\mathrm{s}}=4 \pi R^{2} \Delta_{\mathrm{s}}$, in which neither the stress-free volume nor Eshelby's operations are introduced.

\section{Spherical crack source}

Next, we consider the spherical crack source (S2) of Müller (2001) in an infinite medium. The boundary conditions at $r=R$ are

$$
\begin{aligned}
u_{\mathrm{r}}^{e}-u_{\mathrm{r}}^{i} & =\frac{1}{R^{2}} B-R A=D_{\mathrm{s}}, \\
\sigma_{\mathrm{rr}}^{e}-\sigma_{\mathrm{rr}}^{i} & =-\frac{4 \mu}{R^{3}} B-(3 \lambda+2 \mu) A=0,
\end{aligned}
$$

where $u_{\mathrm{r}}^{i}$ and $\sigma_{\mathrm{rr}}^{i}$ are the radial displacement and radial stress in region 1, respectively. Then, Müller (2001) obtained

$$
\begin{aligned}
d_{\mathrm{s}} & =\frac{4 \mu D_{\mathrm{s}}}{3(\lambda+2 \mu)}, \\
\Delta_{\mathrm{s}} & =\frac{(\lambda+2 \mu / 3) D_{\mathrm{s}}}{(\lambda+2 \mu)} .
\end{aligned}
$$

Using volume $\Delta V_{\mathrm{S}}=4 \pi R^{2} \Delta_{\mathrm{S}}$, the radial displacement is written as

$$
u_{\mathrm{r}}^{e}=\frac{\Delta V_{\mathrm{s}}}{4 \pi r^{2}},
$$

where

$$
\Delta V_{\mathrm{s}}=\frac{(\lambda+2 \mu / 3)}{(\lambda+2 \mu)} \Delta V_{\mathrm{D}}
$$

Here, $\Delta V_{\mathrm{D}}=4 \pi R^{2} D_{\mathrm{s}}$.

\section{Isotropic source}

We next consider the displacement field due to an isotropic source represented by three vector dipoles (S3). The static displacement field due to the moment tensor $M_{p q}$ is given as

$$
\begin{gathered}
u_{\mathrm{i}}=\frac{1}{8 \pi \mu r^{2}}\left[\frac{\lambda+\mu}{\lambda+2 \mu}\left(3 \gamma_{i} \gamma_{p} \gamma_{q}-\left(\gamma_{i} \delta_{p q}+\gamma_{p} \delta_{i q}+\gamma_{q} \delta_{i p}\right)\right)\right. \\
\left.+2 \gamma_{q} \delta_{i p}\right] M_{p q},
\end{gathered}
$$

where $\gamma_{i}$ is the directional cosine and $\delta_{i j}$ is the Kronecker delta (Aki and richards 2002). Using the moment tensor for the isotropic source in Equation 7, we obtain the radial displacement due to the isotropic source in spherical coordinates as follows:

$$
u_{\mathrm{r}}=\frac{M_{0}^{\mathrm{Iso}}}{4 \pi(\lambda+2 \mu) r^{2}} .
$$

For an isotropic source represented by three tensile cracks (S4), Equations 8 and 27 yield

$$
u_{\mathrm{r}}=\frac{(\lambda+2 \mu / 3)}{4 \pi(\lambda+2 \mu)} \frac{\Delta V_{\mathrm{i}}}{r^{2}} .
$$

(b) $S 4$ Infinite medium $\frac{\Delta V_{s}}{4 \pi r^{2}} \underset{F 1}{2 \pi(\lambda+\mu)} \frac{\Delta V_{s}}{r^{2}}$
(a) 1 Half-space
Figure 4 Static displacement fields. These fields are due to (a) a spherical source (S1) and (b) an isotropic source (S4) in an infinite medium and
in a half-space.


Following Okada (1992), we find that the radial displacement on the free surface at the distance $r$ from the isotropic source in a half-space is given as

$$
u_{\mathrm{r}}=\frac{(\lambda+2 \mu / 3)}{2 \pi(\lambda+\mu)} \frac{\Delta V_{\mathrm{i}}}{r^{2}} .
$$

Comparing Equation 28 with Equation 29, we find, as in the spherical source or Mogi model (Equations 15 and 17), that they differ by a factor of $2(\lambda+2 \mu) /(\lambda+\mu)$ (Figure $4 \mathrm{~b})$.

\section{Eshelby spherical source}

We finally consider the displacement field due to an Eshelby spherical source (S5). Before the release of the applied traction, the radial stress in region 1 is given as

$$
\sigma_{\mathrm{rr}}^{i}=-(\lambda+2 \mu / 3) \frac{3 \Delta a}{R} .
$$

In the equilibrium state after the traction release, the displacements and stresses at $r=R$ are given as

$$
\begin{gathered}
u_{\mathrm{r}}^{i}=u_{\mathrm{r}}^{e}=\Delta_{\mathrm{s}}, \\
\sigma_{\mathrm{rr}}^{i}=\sigma_{\mathrm{rr}}^{e}=-\Delta P .
\end{gathered}
$$

Using these boundary conditions, we find that

$$
u_{\mathrm{r}}^{e}=\frac{\Delta V_{\mathrm{s}}}{4 \pi r^{2}},
$$

where

$$
\Delta V_{\mathrm{s}}=\frac{(\lambda+2 \mu / 3)}{(\lambda+2 \mu)} \Delta V_{\mathrm{f}}
$$

Here, $\Delta V_{\mathrm{f}}=4 \pi R^{2} \Delta a$. Comparing Equation 34 with Equation 25, we see that $D_{\mathrm{s}}=\Delta a$.

Instead of applying the stress-free strain, we may apply a stress or pressure $(\delta P)$ to the removed source volume without deforming it (strain-free stress) (S6, Figure 2b). We put the stressed volume back in the hole, weld the material across the cut, and release the stress, which results in the volume source expanding to $R+\Delta_{\mathrm{s}}$. Before the release of the stress, the radial stress in region 1 is given as

$$
\sigma_{\mathrm{rr}}^{i}=-\delta P .
$$

The boundary conditions after the stress release are the same as those for the stress-free strain (Equations 31 and 32 ), and so we find that the displacement field due to this source is

$$
u_{\mathrm{r}}^{e}=\frac{\Delta V_{\mathrm{s}}}{4 \pi r^{2}},
$$

where

$$
\Delta V_{\mathrm{s}}=\frac{4 \pi R^{3}}{3(\lambda+2 \mu)} \delta P .
$$

We note that $\delta P$ is the applied pressure, which may correspond to the stress glut (Backus and Mulcahy 1976), and $\Delta P$ is the pressure after the equilibrium state.

\section{Sources in a bimaterial medium}

We consider a bimaterial medium, in which Lamés constants are $\lambda^{\prime}$ and $\mu^{\prime}$ in region 1 and $\lambda$ and $\mu$ in region 2 . This medium may be viewed as a model of a magma or hydrothermal reservoir, and its source representations are critically important to understand volcanic processes.

For a spherical source (S1), no assumption is made on region 1 , and the boundary conditions at $r=R$ in a bimaterial medium are given as those in a homogeneous medium (Equations 12 and 13). Therefore, the displacement field is the same as in a homogeneous medium given in Equation 15.

For an isotropic source, three vector dipoles (S3) can be represented as three tensile cracks (S4). For the moment tensor of each tensile crack, no assumption is made for the material inside a tensile crack, and we may not able to define an isotropic source in a bimaterial medium.

For a spherical crack source (S2), the boundary conditions at $r=R$ in a bimaterial medium may be given as

$$
\begin{aligned}
u_{\mathrm{r}}^{e}-u_{\mathrm{r}}^{i} & =\frac{1}{R^{2}} B-R A=D_{\mathrm{s}}^{\prime}, \\
\sigma_{\mathrm{rr}}^{e}-\sigma_{\mathrm{rr}}^{i} & =-\frac{4 \mu}{R^{3}} B-\left(3 \lambda^{\prime}+2 \mu^{\prime}\right) A=0,
\end{aligned}
$$

where $D_{\mathrm{s}}^{\prime}=d_{\mathrm{s}}^{\prime}+\Delta_{\mathrm{s}}$. Here, $d_{\mathrm{s}}^{\prime}$ is the inward crack wall displacement in region 1 . We then obtain

$$
u_{\mathrm{r}}^{e}=\frac{\Delta V_{\mathrm{s}}}{4 \pi r^{2}}
$$

and

$$
\Delta V_{\mathrm{s}}=\frac{\left(\lambda^{\prime}+2 \mu^{\prime} / 3\right)}{\left(\lambda^{\prime}+2 \mu^{\prime} / 3+4 \mu / 3\right)} \Delta V_{\mathrm{D}}^{\prime},
$$

where $V_{\mathrm{D}}^{\prime}=4 \pi R^{2} D_{\mathrm{s}}^{\prime}$.

For an Eshelby spherical source with stress-free strain (S5), the source volume of region 1 expands to $R+\Delta a^{\prime}$, and the radial stress in region 1 before the release of the applied traction may be given as

$$
\sigma_{\mathrm{rr}}^{i}=-\left(\lambda^{\prime}+2 \mu^{\prime} / 3\right) \frac{3 \Delta a^{\prime}}{R} .
$$

The boundary conditions at $r=R$ are the same as those in a homogeneous medium (Equations 31 and 32), and so we have

$$
u_{\mathrm{r}}^{e}=\frac{\Delta V_{\mathrm{s}}}{4 \pi r^{2}}
$$

and

$$
\Delta V_{\mathrm{s}}=\frac{\left(\lambda^{\prime}+2 \mu^{\prime} / 3\right)}{\left(\lambda^{\prime}+2 \mu^{\prime} / 3+4 \mu / 3\right)} \Delta V_{\mathrm{f}}^{\prime},
$$

where $\Delta V_{\mathrm{f}}^{\prime}=4 \pi R^{2} \Delta a^{\prime}$. 
For an Eshelby spherical source with strain-free stress $\delta P^{\prime}(\mathrm{S} 6)$, we obtain

$$
u_{\mathrm{r}}^{e}=\frac{\Delta V_{\mathrm{s}}}{4 \pi r^{2}},
$$

and

$$
\Delta V_{\mathrm{s}}=\frac{4 \pi R^{3}}{3\left(\lambda^{\prime}+2 \mu^{\prime} / 3+4 \mu / 3\right)} \delta P^{\prime} .
$$

\section{Results and discussion}

We summarize the radial displacement fields due to the sources examined above in Figure 5. Except for an isotropic source (Figure $5 \mathrm{c}, \mathrm{d}$ ), the displacement fields are given as $\Delta V_{\mathrm{s}} /\left(4 \pi r^{2}\right)$. Let us first consider the relationship between a spherical source (S1, Figure 5a) and an isotropic source represented by three vector dipoles (S3, Figure 5c). The displacement fields of the spherical source and isotropic source are given as

$$
\begin{aligned}
& u_{\mathrm{r}}^{e}=\frac{\Delta V_{\mathrm{s}}}{4 \pi r^{2}}, \\
& u_{\mathrm{r}}=\frac{M_{0}^{\text {Iso }}}{4 \pi(\lambda+2 \mu) r^{2}},
\end{aligned}
$$

respectively. The equivalence of these displacements at $r>R$ provides the following relation:

$$
\Delta V_{\mathrm{s}}=\frac{M_{0}^{\text {Iso }}}{(\lambda+2 \mu)} \text {. }
$$

For an isotropic source represented by three tensile cracks (S4), we obtain Equation 8, i.e.,

$$
M_{0}^{\text {Iso }}=(\lambda+2 \mu / 3) \Delta V_{\mathrm{i}} .
$$

The relation of Equation 49 is also held between a spherical crack source (S2) and an isotropic source (S3) and between an Eshelby spherical source (S5 and S6) and an isotropic source (S3) (see Figure 5). Then, using Equations 25 and 34, we find that the following relation is held for a spherical crack source (S2) and an Eshelby spherical source with stress-free strain (S5):

$$
M_{0}^{\text {Iso }}=(\lambda+2 \mu / 3) \Delta V_{\mathrm{f}},
$$

where $\Delta V_{\mathrm{f}}=4 \pi R^{2} \Delta a=4 \pi R^{2} D_{\mathrm{s}}$. We see from Equations 50 and 51 that $\Delta V_{\mathrm{i}}=\Delta V_{\mathrm{f}}$ : the sum of three tensile crack volumes is equal to a spherical crack volume or stress-free volume. Therefore, the two seismic moment equations (Equations 1 and 2) can be understood as follows: the first equation from the equivalence of the displacement fields

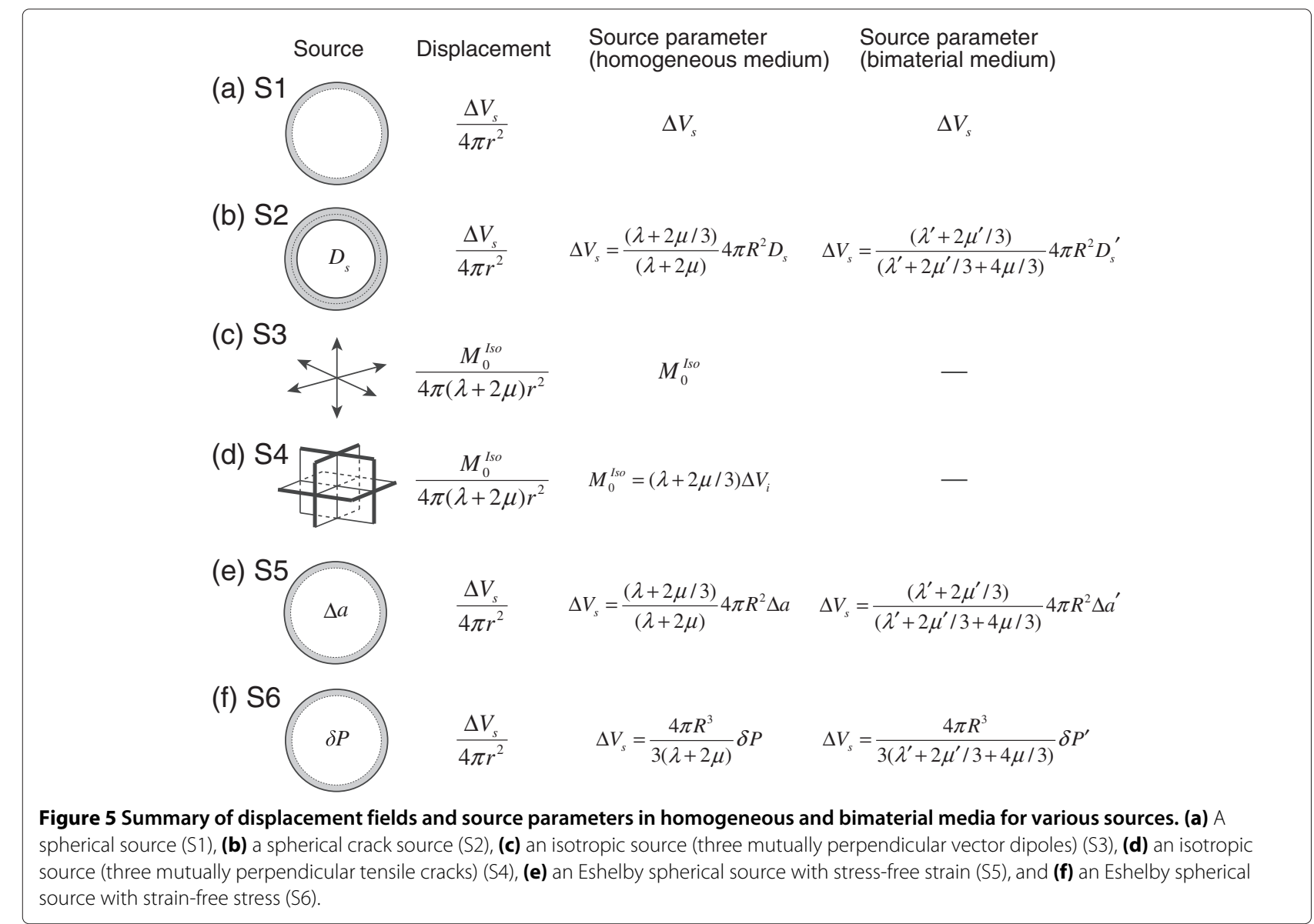


due to a spherical source (S1) and an isotropic source (S3) (Equation 49) and the second equation from the equivalence of three tensile crack volumes and a spherical crack volume or stress-free volume (Equations 50 and 51). The first equation can be used to estimate the actual volume from the seismic moment of an isotropic source (S3).

As shown in Subsection 'Sources in a bimaterial medium', no assumption is made on region 1 for a spherical source ( $\mathrm{S} 1)$, and its source parameter is given as $\Delta V_{\mathrm{s}}$ in both homogeneous and bimaterial media (Figure 5a). Thus, we may use Equation 49 with $M_{0}^{\text {Iso }}$ in a homogeneous medium to estimate $\Delta V_{\mathrm{s}}$, regardless of the assumption on the medium.

It is interesting to note that we may be able to estimate a spherical crack volume or stress-free volume (Equation 51) that cannot be observed. This estimation, however, is based on our interpretation of the source process in region 1. For example, if we consider an Eshelby spherical source with strain-free stress (S6, Figure 5f), we obtain

$$
M_{0}^{\text {Iso }}=\frac{4 \pi R^{3}}{3} \delta P
$$

which gives an estimation of $\delta P$ in region 1 . If we assume a bimaterial medium for a spherical crack source (S2) and consider an Eshelby spherical source with stress-free strain (S5), we have

$$
M_{0}^{\text {Iso }}=\frac{(\lambda+2 \mu)\left(\lambda^{\prime}+2 \mu^{\prime} / 3\right)}{\left(\lambda^{\prime}+2 \mu^{\prime} / 3+4 \mu / 3\right)} \Delta V_{\mathrm{f}}^{\prime},
$$

where $\Delta V_{\mathrm{f}}^{\prime}=4 \pi R^{2} D_{\mathrm{s}}^{\prime}=4 \pi R^{2} \Delta a^{\prime}$. For an Eshelby spherical source with strain-free stress (S6) in a bimaterial medium, we obtain

$$
M_{0}^{\text {Iso }}=\frac{4 \pi(\lambda+2 \mu) R^{3}}{3\left(\lambda^{\prime}+2 \mu^{\prime} / 3+4 \mu / 3\right)} \delta P^{\prime} .
$$

These results suggest that the source estimation in region 1 depends on our interpretation, whereas we can uniquely estimate the source parameter of $\Delta V_{\mathrm{S}}$ in region 2 from $M_{0}^{\text {Iso }}$.

In volcano seismological studies, waveform inversions have been performed to determine moment tensor solutions. If one interprets the isotropic source determined by waveform inversion to be spherical, then Equation 49 can be used to estimate the actual volume $\Delta V_{\mathrm{s}}$. In volcano geodetic studies, the Mogi model has been used to interpret volcano deformations. Because the Mogi model is based on a spherical source, $\Delta V_{\mathrm{s}}$ is estimated directly. On the other hand, Okada (1992) model uses an isotropic source. Thus, if one estimates the seismic moment of an isotropic source by using Okada's model, the relation given in Equation 49 should be used to estimate the volume change due to the corresponding spherical source or Mogi model.

We see that the displacement fields due to the described sources in an infinite medium are all expressed as a function of $r^{-2}$ (Figure 5). The divergence of such a function is zero, which means that a source volume change must be conserved everywhere in an infinite medium. For all the sources except for an isotropic source, we obtain the following relation in an infinite medium:

$$
4 \pi r^{2} u_{\mathrm{r}}=\Delta V_{\mathrm{s}}
$$

The left-hand side represents the volume change of a sphere with an arbitrary radius $r$ centered at the source, which is equal to $\Delta V_{\mathrm{s}}$. The source volume change in this displacement field is, thus, conserved in an arbitrary subspace enclosing the source. For an isotropic source represented by three tensile cracks (S4), we obtain the following relation:

$$
4 \pi r^{2} u_{\mathrm{r}}=\frac{(\lambda+2 \mu / 3)}{(\lambda+2 \mu)} \Delta V_{\mathrm{i}}
$$

This relation indicates that the source volume change is not conserved. The non-conserved volume may be attributed to compressed volumes in regions close to the cracks (Figure 6). The non-conserved volume is equal to the spherical crack volume or stress-free volume, although the reason why these volumes are equal is not clear. We note that the conservation of a source volume does not hold for displacement fields in a half-space because of the effect of the free surface.

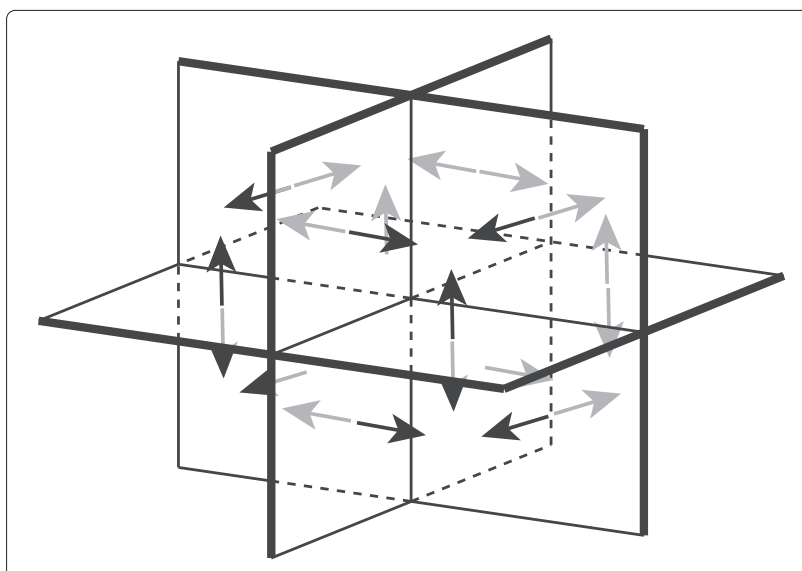

Figure 6 Force systems for three mutually perpendicular tensile cracks. 
Let us further consider a spherical crack source in a homogeneous medium with the following boundary conditions at $r=R$ :

$$
\begin{aligned}
u_{\mathrm{r}}^{e} & =\Delta_{\mathrm{s}} \\
\sigma_{\mathrm{rr}}^{e} & =-\Delta P \\
u_{\mathrm{r}}^{i} & =-d_{\mathrm{s}} \\
\sigma_{\mathrm{rr}}^{i} & =\Delta \bar{P} \\
u_{\mathrm{r}}^{e}-u_{\mathrm{r}}^{i} & =\frac{1}{R^{2}} B-R A=\Delta_{\mathrm{s}}+d_{\mathrm{s}} \\
\sigma_{\mathrm{rr}}^{e}-\sigma_{\mathrm{rr}}^{i} & =-\frac{4 \mu}{R^{3}} B-(3 \lambda+2 \mu) A=-(\Delta P+\Delta \bar{P})
\end{aligned}
$$

These boundary conditions represent the displacement discontinuity $\Delta_{\mathrm{s}}+d_{\mathrm{s}}$ and the stress or traction discontinuity $-(\Delta P+\Delta \bar{P})$ along the spherical surface. Using Equations 61 and 62, we obtain

$u_{\mathrm{r}}^{e}=\left[\frac{R}{3(\lambda+2 \mu)}(\Delta P+\Delta \bar{P})+\frac{(\lambda+2 \mu / 3)}{(\lambda+2 \mu)}\left(\Delta_{\mathrm{s}}+d_{\mathrm{s}}\right)\right]\left(\frac{R}{r}\right)^{2}$.

This equation represents the displacement field due to a generalized spherical crack source having both displacement and traction discontinuities. Using Equations 59 and 60 , we obtain

$$
\Delta \bar{P}=-(3 \lambda+2 \mu) \frac{d_{\mathrm{s}}}{R} .
$$

Substituting Equation 64 into Equation 63, we obtain

$$
u_{\mathrm{r}}^{e}=\left[\frac{R}{3(\lambda+2 \mu)} \Delta P+\frac{(\lambda+2 \mu / 3)}{(\lambda+2 \mu)} \Delta_{\mathrm{s}}\right]\left(\frac{R}{r}\right)^{2} .
$$

From Equations 57 and 58, we see that Equation 65 becomes Equation 15 for the displacement field due to a spherical source. Therefore, Equation 65 may be regarded as the general form of the equation for the displacement field due to a spherical source represented by $\Delta P$ and $\Delta_{\mathrm{s}}$.

\section{Conclusions}

We systematically examined the following seismic sources and their static displacement fields: a spherical source (radius $R$ ) with outward radial expansion $\left(R+\Delta_{\mathrm{S}}\right)(\mathrm{S} 1)$, a spherical crack source with outward and inward crack wall motions along the spherical surface with displacement discontinuity $D_{\mathrm{s}}(\mathrm{S} 2)$, an isotropic source represented by three mutually perpendicular vector dipoles (S3) or three tensile cracks (S4), and an Eshelby spherical source undergoing a transformational expansion $(R+\Delta a)$ (S5) or strainfree stress $(\delta P)(\mathrm{S} 6)$. Our examinations show that the static radial displacement fields due to these sources except for an isotropic source are given as $\Delta V_{\mathrm{s}} /\left(4 \pi r^{2}\right)$, where
$\Delta V_{\mathrm{s}}=4 \pi R^{2} \Delta_{\mathrm{s}}$ is the actual volume. The equivalence of these fields and that due to an isotropic source (S3) gives $\Delta V_{\mathrm{s}}=M_{0}^{\text {Iso }} /(\lambda+2 \mu)$, where $M_{0}^{\text {Iso }}$ is the seismic moment of an isotropic source. The actual volume $\Delta V_{\mathrm{s}}$ may be uniquely determined from $M_{0}^{\text {Iso }}$ in homogeneous and bimaterial media. We see that the source volume change for an isotropic source defined by three tensile cracks (S4) is equal to the spherical crack volume $\left(4 \pi R^{2} D_{\mathrm{S}}\right)$ and stress-free volume $\left(4 \pi R^{2} \Delta a\right)$. We then obtain $M_{0}^{\text {Iso }}=(\lambda+2 \mu / 3) \Delta V_{\mathrm{f}}$, where $\Delta V_{\mathrm{f}}$ is the spherical crack or stress-free volume. This relation depends on our interpretation of the source process. If we consider an Eshelby spherical source with strain-free stress (S6), $M_{0}^{\text {Iso }}$ is related to $\delta P$. We obtain a different estimation of $\Delta V_{\mathrm{f}}$ or $\delta P$ in a bimaterial medium. This study provides a unified view for quantifying a spherical source using the seismic moment of an isotropic source determined from waveform inversion analysis in volcano seismological studies.

\section{Competing interests}

The authors declare that they have no competing interests.

\section{Authors' contributions}

HK developed the main framework of the study and drafted the manuscript. All authors carried out the theoretical considerations and read and approved the final manuscript.

\section{Acknowledgements}

We thank Mare Yamamoto, Minoru Takeo, and Shiro Hirano for their useful discussions. This work was supported by the Japan Science and Technology Agency (JST).

\section{Author details}

${ }^{1}$ Graduate School of Environmental Studies, Nagoya University, Furo-cho, Chikusa-ku, Nagoya 464-8601, Japan. ${ }^{2}$ Earthquake Research Institute, The University of Tokyo, 1-1-1 Yayoi, Bunkyo-ku, Tokyo 113-0032, Japan.

Received: 14 December 2013 Accepted: 25 March 2014

Published: 7 April 2014

\section{References}

Aki K, Richards PG (2002) Quantitative seismology, 2nd edn. University Science Books, Sausalito

Ampuero JP, FA Dahlen (2005) Ambiguity of the moment tensor. Bull Seism Soc Am 95: 390-400

Backus G, Mulcahy M (1976) Moment tensors and other phenomenological descriptions of seismic sources-I. Continuous displacements. Geophys J R Astr Soc 46: 341-361

Chouet B (1996) A seismic model for the source of long-period events and harmonic tremor. In: Gasparini P, Scarpa R, Aki K (eds) Volcanic seismology. Springer, Berlin, pp 133-156

Chouet B (2013) Introduction to quantitative volcano seismology: fluid-driven sources. In: Fragents SA, Gregg, TKP, Lopes, RMC (eds) Modeling volcanic processes the physics and mathematics of volcanism. Cambridge University Press, Cambridge, pp 331-358

Dreger D, Tkalčić H, Johnston M (2000) Dilational processes accompanying earthquakes in the Long Valley Caldera. Science 288: 122-125

Eshelby JD (1957) The determination of the elastic field of an ellipsoidal inclusion, and related problems. Proc Roy Soc Ser A 241: 376-396

Fukuyama E, Takeo M (1987) Analysis of the near-field seismogram observed during the eruption of Izu-Oshima Volcano on November 16. Bull Volcanol Soc Jpn 35: 283-297

Julian BR, Miller AD, Foulger GR (1998) Non-double-couple earthquakes 1. Theory. Rev Geophys 36: 525-549 
Kanamori H, Given J, Lay T (1980) Analysis of seismic body waves excited by the Mount St. Helens eruption of May 18, J Geophys Res 89: 1856-1866 Kawakatsu H, Yamamoto M (2007) Volcano seismology In: Kamanori H (ed) Treatise on geophysics volume 4-earthquake seismology. Elsevier, Amsterdam, pp 389-420

Kumagai H (2009) Source quantification of volcano seismic signals. In: Mayers RA (ed) Encyclopedia of complexity and systems science. Springer, New York

Kumagai H, Placios P, Ruiz M, Yepes H, Kozono T (2011) Ascending seismic source during an explosive eruption at Tungurahua volcano. Ecuador Geophys Res Lett 38. L01306 doi:10.1029/2010GL045944

Legrand D, Kaneshima S, Kawakatsu H (2000) Moment tensor analysis of near-field broadband waveforms observed at Aso volcano, Japan. J Volcanol Geotherm Res 101: 155-169

Maeda M, Takeo M (2011) Take very-long-period pulses at Asama volcano, central Japan, inferred from dense seismic observations. Geophys J Int 185: 265-282

Mogi K (1958) Relation between the eruptions of various volcanoes and the deformations of the ground surfaces around them. Bull Earthq Res Inst Univ Tokyo 36: 99-134

Müller G (2001) Volume change of seismic. Bull Seism Soc Am 91: 880-884

Nishimura T, Iguchi M (2011) Volcanic earthquakes and tremor in Japan. Kyoto University Press, Kyoto

Ohminato T, Takeo M, Kumagai H, Yamashina T, Oikawa J, Koyama E, Tsuji H, Urabe T (2006) Vulcanian eruptions with dominant single force components observed during the Asama 2004. volcanic activity in Japan Earth Planets Space 58: 583-593

Okada Y (1992) Internal deformation due to shear and tensile faults in a half-space. Bull Seism Soc Am 82: 1018-1040

Richards PG, Kim WY (2005) Equivalent volume sources for explosions at depth: theory and observations 95: 401-407

Sezawa K (1931) The plastico-elastic deformation of a semi-infinite solid body due to an internal force 9: 398-406

Tameguri T, Iguchi M, Ishihara K (2002) Mechanism of explosive eruptions from moment tensor analyses of explosion earthquakes at Sakurajima volcano, Japan. Bull Volcanol Soc Jpn 47: 197-216

Uhira K, Takeo M (1994) The source of explosive eruptions of Sakurajima volcano, Japan. J Geophys Res 99: 17775-17789

Wielandt E (2003) On the relationship between seismic moment and source volume. http://www.geophys.uni-stuttgart.de/ erhard/skripte/ew/ isomoment/ Accessed May 302003

doi:10.1186/1880-5981-66-7

Cite this article as: Kumagai et al: Seismic moment and volume change of a spherical source. Earth, Planets and Space 2014 66:7.

\section{Submit your manuscript to a SpringerOpen ${ }^{\circ}$ journal and benefit from:}

- Convenient online submission

- Rigorous peer review

- Immediate publication on acceptance

- Open access: articles freely available online

- High visibility within the field

- Retaining the copyright to your article

Submit your next manuscript at $\boldsymbol{\nabla}$ springeropen.com 